\title{
Neurotoxocarosis: marked preference of Toxocara canis for the cerebrum and T. cati for the cerebellum in the paratenic model host mouse
}

\author{
Elisabeth Janecek ${ }^{1}$, Andreas Beineke ${ }^{2}$, Thomas Schnieder ${ }^{1 \wedge}$ and Christina Strube ${ }^{1 *}$
}

\begin{abstract}
Background: Infective larvae of the worldwide occurring zoonotic roundworm T. canis exhibit a marked affinity to the nervous tissues of paratenic hosts. In humans, most cases of neurotoxocarosis are considered to be caused by larvae of T. canis as T. cati larvae have rarely been found in the CNS in previous studies. However, direct comparison of studies is difficult as larval migration depends on a variety of factors including mouse strains and inoculation doses. Therefore, the present study aims to provide a direct comparison of both roundworm species in mice as a model for paratenic hosts with specific focus on the CNS during the acute and chronic phase of disease to provide a basis for further studies dealing with neurotoxocarosis.

Methods: C57B//6J mice were infected with 2000 embryonated T. canis and T. cati eggs, respectively as well as Balb/c mice infected with $T$. cati eggs only. On 8 time points post infection, organs were removed and microscopically examined for respective larvae. Special focus was put on the CNS, including analysis of larval distribution in the cerebrum and cerebellum, right and left hemisphere as well as eyes and spinal cord. Additionally, brains of all infection groups as well as uninfected controls were examined histopathologically to characterize neurostructural damage.
\end{abstract}

Results: Significant differences in larval distribution were observed between and within the infection groups during the course of infection. As expected, significantly higher recovery rates of $T$. canis than $T$. cati larvae were determined in the brain. Surprisingly, significantly more T. canis larvae could be found in cerebra of infected mice whereas T. cati larvae were mainly located in the cerebellum. Structural damage in brain tissue could be observed in all infection groups, being more severe in brains of $T$. canis infected mice.

Conclusions: The data obtained provides an extensive characterization of migrational routes of $T$. canis and $T$. cati in the paratenic host mouse in direct comparison. Even though to a lesser extent, structural damage in the brain was also caused by T. cati larvae and therefore, the potential as pathogenic agents should not be underestimated.

Keywords: Toxocara canis, Toxocara cati, Roundworms, Larval migration, Neurotoxocarosis, Zoonotic helminths

\section{Background}

Toxocara spp. are worldwide occurring helminths of carnivores with high zoonotic potential [1]. Larvae undergo a full development into the adult stage in the definitive host "carnivore" which sheds large amounts of eggs into the environment. Under suitable environmental conditions, the infective third stage larva develops in the egg $[2,3]$,

\footnotetext{
* Correspondence: christina.strube@tiho-hannover.de

Deceased

'Institute for Parasitology, University of Veterinary Medicine Hannover,

Buenteweg 17, 30559 Hannover, Germany

Full list of author information is available at the end of the article
}

which may be taken up by paratenic hosts, including humans. After ingestion, larvae hatch in the small intestine, migrate through the paratenic host's organs and persist as the infectious stadium in different tissues for prolonged periods of time. Additionally, consumption of infected paratenic hosts may result in infection as persisting larvae are reactivated and continue migration [4-8]. Migrational behaviour and structural damage caused by larvae of the canine roundworm $T$. canis have been characterized extensively in several paratenic hosts finding a strong neural affinity whereas migrational behaviour and 
damages caused by $T$. cati larvae have been neglected [9-11]. Even though both roundworm species share antigenic fractions and behave similarly in animal models after hatching, the zoonotic potential of $T$. cati is often underestimated [11]. It is assumed, that most human cases of neurotoxocarosis result from infection with $T$. canis as no reliable differentiation between $T$. canis and $T$. cati larvae is established and previous studies mainly revealed accumulation of $T$. canis larvae in brains of infected mice [9,12-14]. In contrast, few studies report accumulation of $T$. cati larvae mainly in the muscle and only low larval numbers in the brain [15-18].

Human neurotoxocarosis assumingly caused by $T$. canis larvae has been described in several cases; however, occurrence is rather rare. Commonly described clinical symptoms in humans have been behavioral disorders, focal or generalized seizures, ataxia, sensory disturbances, brain infarcts as well as urinary retention [19-23]. Sporadically, cognitive dysfunctions and reduced ability of long and short term memory have been reported $[20,22,24]$. Infrequently, $T$. cati infection has been suggested with the patient presenting symptoms like mild spastic paraplegia, superficial sensory impairment, urinary retention and slightly brisk deep tendon reflexes [25].

Mice are a valuable model for characterization of infection progress as they present similar clinical manifestations compared to humans. Behavioural changes like abnormal social behaviour, increase of immobility, decrease of exploratory behaviour as well as impairment of learning and memory have been observed in mice infected with T. canis [26-29]. Furthermore, central nervous symptoms such as dullness, paresis and tremor were described accompanied by pathological findings like demyelination, focal malacia and mixed cell infiltration $[27,30]$. As migrational behaviour differs between mouse strains and infection doses [30,31], direct comparison of both roundworm species under equal experimental parameters is essential. The chronic course of a $T$. cati infection in comparison to T. canis infection focusing on the CNS has not been clearly characterized yet. Therefore, the aim of the present study was to directly compare migration routes of $T$. canis and T. cati in $\mathrm{C} 57 \mathrm{Bl} / 6 \mathrm{~J}$ (B6) mice as well as T. cati in Balb/c mice and observe brain tropism for both species as well as possible neuropathological changes resulting from migration to the brain. This provides a valuable model to extrapolate results to human neurotoxocarosis and serves as a foundation for further molecular studies.

\section{Methods}

\section{Animal models and infectious material}

Animal experiments were permitted by the ethics commission of the Lower Saxony State Office for Consumer Protection and Food Safety under reference numbers 33.14-42502-04-11/0336 and 33.9-42502-05-01A038. Mouse strains $\mathrm{C} 57 \mathrm{Bl} / 6$ )RccHsd mice as well as Balb/cOlaHsd mice (Harlan Laboratories, Horst, Netherlands) were used as model organisms. Infective material consisted of $T$. canis and $T$. cati eggs obtained from feces of experimentally infected dogs and cats, respectively. Eggs were purified by combined sedimentation- flotation method with subsequent rinsing in tap water. Eggs were incubated at $25^{\circ} \mathrm{C}$ for 4 weeks for embryonation.

Mice were infected orally with 2000 embryonated $T$. canis or T. cati eggs, respectively in a total volume of $0.5 \mathrm{ml}$ tap water at five weeks of age. Infection groups were divided into 64 (32 male/32 female) Balb/c mice infected with T. cati, 64 (32 male/32 female) C57Bl/6J (B6) mice infected with $T$. cati and 64 (32 male/32 female) B6 mice infected with $T$. canis. As the literature provides extensive data concerning $T$. canis migrational routes in Balb/c mice, this strain was omitted for $T$. canis as sufficient data is available for comparison. A placebo group of $16 \mathrm{~B} 6$ mice was given $0.5 \mathrm{ml}$ tab water. At day 2, 7, 14, 21, 28, 35, 42 and 98 post infection (pi), eight mice per infection group as well as two control mice were euthanized by cervical dislocation. Lungs, liver, heart, kidneys, spleen, muscle, eyes, spinal cord and the brain were removed and stored individually at $-80^{\circ} \mathrm{C}$ until digestion. Out of the eight brains per group, two were used for subsequent histopathological examination and six brains were divided into cerebrum, cerebellum and subdivided into left and right hemisphere before freezing at $-80^{\circ} \mathrm{C}$.

\section{Pathological examination}

Organs were examined macroscopically at dissections. For histopathological examination, two brains (one male and one female mouse) of each group were fixed in 10\% formalin for at least three days. Brains were cut coronally at the bregma zero coordinate as well as about $5 \mathrm{~mm}$ to the left and 3 and $7 \mathrm{~mm}$ to the right of the bregma zero coordinate according to the mouse brain atlas [32] and embedded in paraffin. Embedded sections were cut to obtain $2 \mu \mathrm{m}$ thick slices, which were stained with haematoxylin and eosin (H \& E stain).

\section{Artificial digestion}

Determination of the larval counts in the organs was accomplished by artificial digestion and subsequent microscopical count. Organs were thawed and cut into small pieces which were digested in a solution containing $300 \mathrm{mM} \mathrm{HCl}$ (Carl Roth GmbH, Karlsruhe, Germany) and $1 \%$ pepsin (Merck, Darmstadt, Germany). Incubation occurred at $37^{\circ} \mathrm{C}$ in a water bath while constantly stirring for approximately 2 hours. Solution was centrifuged at $3000 \times g$ for $30 \mathrm{~min}$. The supernatant was discarded and the pellet microscopically examined for larval counts. 


\section{Statistical analysis}

Statistical analyses were carried out using SAS software (version 9.3). Analysis of single organs over time and between groups was conducted using 2-way ANOVA with subsequent Ryan-Einot-Gabriel-Welsch multiple range test to control experimentwise error rate. Comparisons of larval distribution between individual organs were made using least significant difference (LSD) post hoc tests.

\section{Results}

\section{General larval distribution}

Artificial digestion revealed statistically significant differences between $T$. canis and $T$. cati larval migration. On day 2 pi, significantly more $T$. canis than $T$. cati larvae were detected in the liver. In contrast, significantly less T. canis larvae could be detected in lungs at this time point- with significantly more $T$. cati larvae in lungs of B6 than in Balb/c mice. Looking at the course of infection within infection groups, larval counts in liver and lungs decreased significantly after day 2 in Balb/c T. cati infected mice. Larval counts in lungs and livers of B6 T. cati and in livers of $T$. canis infected mice decreased significantly starting day 2 pi. Larval counts in liver and lungs of all infection groups remained on a low level during the later time points. During all time points post infection, significantly more $T$. cati than $T$. canis larvae could be recovered from the muscle whereas no significant differences between muscle larvae recovery rates were observed for Balb/c and B6 T. cati infected mice.

In the heart, kidneys and spleen, larvae could be recovered only sporadically and in very low numbers, rarely exceeding one larva on average, in all infection groups during the entire study. Therefore, they were excluded from statistical analysis.

A detailed overview of larval numbers as well as recovery rates in the examined organs is given in Table 1. Overall, the total number of recovered larvae was much higher in $T$. cati infected mice whereas only very few $T$. canis larvae could be recovered based on the total infection dose. Larval recovery rates for all infection groups during the course of infection are graphically shown in Figure 1. Detailed data concerning statistically significant differences between and within infection groups over the course of infection are shown in Tables 2 and 3.

\section{Larval distribution in the CNS}

Larval distribution in brains revealed statistically significant differences between $T$. canis and $T$. cati infected mice whereas significantly more larval numbers were detected in brains of $T$. canis infected mice with the exception of day 2 pi (cf. Table 2). Within T. cati infection groups, significantly lower larval numbers were recovered from brain compared to muscle tissue for each time point post infection. Detailed data concerning comparison of organs and brain within infection groups including corresponding p-values are provided in Table 4.

On day $2 \mathrm{pi}$, the brain recovery rate (Figure 2) for all infection groups was low resulting on average in $2.4 \%$ and $1.8 \%$ in Balb/c and B6 T. cati infected mice as well as $0.3 \%$ in B6 T. canis infected mice. On day 7 pi, the recovery rate of $T$. cati larvae in Balb/c mice was rather high with $16.5 \%$ when compared to B6 T. cati infected mice (4.5\%); however, no significant difference was observed. In contrast, $46.6 \%$ of recovered $T$. canis larvae were found in the brain at day $7 \mathrm{pi}$. In Balb/c as well as B6 $T$. cati infected mice, recovery rates in the brain remained rather low during the remaining course of infection (4.5\% on average in Balb/c mice and $2.73 \%$ on average in B6 T. cati infected mice). In Balb/c T. cati infected mice, recovery rates differed significantly on day 7 pi vs. day 21 and 28 pi, at which larval counts dropped to a minimum. No significant differences during the course of infection were found in B6 T. cati infected mice. T. canis infected mice revealed a rather high recovery rate in the brain with an average of $32.57 \%$. Significant differences were observed on day 2 pi vs. the succeeding time points, as well as day 7 pi vs. day 28 and 42 pi, respectively. Larval distribution in the cerebrum compared to the cerebellum revealed significant differences between $T$. cati and $T$. canis infected mice with statistically significantly higher larval recovery rates in the cerebra of $T$. canis than in $T$. cati infected mice beginning from day 14 throughout day 98 pi. In contrast, significantly higher recovery rates were observed in cerebella of $T$. cati infected mice on day 28, 35 and 98 pi when compared to T. canis infected mice. Between both $T$. cati infection groups, no statistically significant differences were observed. Larval recovery rates from the cerebellum and cerebrum are graphically displayed in Figures 3 and 4. Concerning larval distribution in the right and left hemisphere, no significant differences were found between left and right parts of the brain within the infection groups at the different time points post infection. Significant differences between infection groups over the course of infection for cerebellum and cerebrum as well as right and left hemisphere are provided in Table 2 .

Analysis of eyes and spinal cords revealed no differences between the infection groups. Only few larvae could be recovered from these organs (cf. Table 5) with larval counts ranging between 0.0 and 1.6 larvae on average during the entire course of infection. Therefore, eyes and spinal cord were excluded from subsequent statistical analysis.

\section{Macroscopical changes and histopathology}

Macroscopical observation of removed organs showed severe hemorrhages in lungs on day $2 \mathrm{pi}$ in Balb/c T. cati infected mice (4/7 mice, $57.14 \%)$ as well as in B6 T. cati 
infected mice (1/8 mice, $12.5 \%)$. No lesions were observed in T. canis infected mice. On day 7 pi, lungs of all infection groups were affected showing severe hemorrhages (Balb/c T. cati infected: $2 / 7$ mice, 28.57\%; B6 T. cati infected: 4/7 mice, 57.14\%; T. canis infected: $3 / 7$ mice, 42.86\%). Lesions were mainly transient and only observed sporadically after day 14 pi. However, in Balb/c T. cati infected mice, hemorrhages in lungs reappeared on day 42 pi (3/8 mice, $37.50 \%)$ and were also observed on day 98 pi (4/5 mice, $80.00 \%)$.

On day $14 \mathrm{pi}, T$. canis infected mice showed petechial hemorrhages on the liver surface and multifocal retractions of the renal parenchyma (5/8 mice, $62.5 \%$, respectively).
Those changes in liver and kidney were not observed in either $T$. cati infection group. Starting day 21 pi until day $42 \mathrm{pi}$, spleen surfaces infrequently showed focal hyperemia in all infection groups.

No changes were observed macroscopically or microscopically on day 2 pi in any of the brains. Starting day 7 pi, severe hemorrhages (Figure 5) were observed macroscopically in the brains of both $T$. canis and T. cati infected mice (Balb/c T. cati infected: 2/7 mice, 28.57\%; B6 T. cati infected: $1 / 7$ mice, $14.29 \%$; B6 T. canis infected: $3 / 7$ mice, $42.86 \%$ ), which were also seen histopathologically (Figure 6a). Hemorrhages were mainly observed in the brain cortex and were more severe in $T$. canis than $T$. cati

Table 1 Larval distribution and recovery rates

\begin{tabular}{|c|c|c|c|c|c|c|c|c|c|}
\hline \multirow[t]{2}{*}{ Organ } & \multirow[t]{2}{*}{ Infection group } & \multicolumn{8}{|c|}{ Day pi } \\
\hline & & 2 & 7 & 14 & 21 & 28 & 35 & 42 & 98 \\
\hline \multirow[t]{6}{*}{ Liver } & T. cati Balb/c & 53.7 & 3.1 & 0.9 & 1.3 & 0.1 & 0.0 & 0.6 & 2.2 \\
\hline & & [24.53] & {$[1.08]$} & {$[1.56]$} & {$[0.57]$} & {$[0.83]$} & {$[0.00]$} & {$[0.53]$} & [0.98] \\
\hline & T. cati B6 & 27.0 & 0.9 & 1.6 & 1.3 & 0.0 & 0.0 & 0.4 & 0.8 \\
\hline & & [21.19] & [1.33] & {$[1.90]$} & {$[0.47]$} & {$[0.00]$} & {$[0.00]$} & {$[0.32]$} & {$[0.35]$} \\
\hline & T. canis B6 & 65.8 & 2.6 & 1.5 & 1.5 & 0.9 & 1.5 & 1.1 & 1.3 \\
\hline & & [89.3] & {$[10.28]$} & [2.69] & [3.33] & {$[1.52]$} & [3.33] & {$[2.10]$} & [3.52] \\
\hline \multirow[t]{6}{*}{ Lung } & T. cati Balb/c & 80 & 1.3 & 0.6 & 0.3 & 0.0 & 0.0 & 0.3 & 0.6 \\
\hline & & [35.97] & {$[0.35]$} & {$[0.5]$} & {$[0.11]$} & {$[0.00]$} & {$[0.00]$} & {$[0.16]$} & [0.29] \\
\hline & T. cati B6 & 49.9 & 15.3 & 0.3 & 5.3 & 0.8 & 0.3 & 0.4 & 0.4 \\
\hline & & [47.38] & {$[22.51]$} & {$[0.14]$} & {$[4.07]$} & {$[0.42]$} & {$[1.79]$} & {$[0.46]$} & {$[0.20]$} \\
\hline & T. canis B6 & 5.6 & 2.4 & 0.3 & 0.4 & 1.0 & 2.3 & 0.8 & 0.1 \\
\hline & & [9.10] & [9.87] & {$[0.40]$} & {$[0.81]$} & {$[1.54]$} & {$[4.31]$} & {$[1.27]$} & {$[0.37]$} \\
\hline \multirow[t]{6}{*}{ Muscle } & T. cati Balb/c & 60.4 & 246.7 & 83.9 & 203.8 & 20.0 & 7.6 & 172.0 & 313.8 \\
\hline & & {$[32.23]$} & [85.39] & [91.62] & [97.53] & [98.33] & {$[96.48]$} & {$[92.53]$} & [95.18] \\
\hline & T. cati B6 & 33.0 & 45.4 & 169.9 & 235.6 & 87.5 & 11.4 & 179.6 & 255.3 \\
\hline & & [26.93] & {$[69.55]$} & [96.89] & {$[92.61]$} & {$[97.68]$} & {$[91.95]$} & {$[97.23]$} & [95.89] \\
\hline & T. canis B6 & 0.4 & 14.3 & 42.4 & 37.0 & 50.4 & 34.0 & 26.4 & 26.8 \\
\hline & & {$[0.49]$} & [39.85] & {$[74.54]$} & {$[72.88]$} & {$[82.83]$} & [59.86] & [51.95] & {$[58.27]$} \\
\hline \multirow[t]{6}{*}{ CNS including eyes } & T. cati Balb/c & 6.8 & 6.2 & 6.1 & 4.3 & 0.2 & 0.7 & 15.3 & 22.9 \\
\hline & & {$[3.65]$} & {$[17.14]$} & {$[6.36]$} & {$[1.79]$} & {$[1.11]$} & {$[4.70]$} & {$[8.50]$} & {$[5.48]$} \\
\hline & T. cati B6 & 4.0 & 3.8 & 3.8 & 7.4 & 1.1 & 0.3 & 6.8 & 9.4 \\
\hline & & {$[2.59]$} & {$[7.77]$} & {$[1.48]$} & {$[3.41]$} & {$[2.52]$} & {$[2.78]$} & {$[2.95]$} & [4.65] \\
\hline & T. canis B6 & 0.3 & 33.9 & 17.4 & 16.2 & 12.5 & 28.7 & 36.3 & 22.9 \\
\hline & & {$[0.31]$} & {$[47.43]$} & {$[29.28]$} & {$[29.22]$} & [17.35] & {$[42.79]$} & {$[57.50]$} & [48.96] \\
\hline \multirow[t]{6}{*}{ Total number of larvae } & T. cati Balb/c & 206.7 & 257.1 & 90.6 & 209.1 & 20.3 & 8.1 & 185.6 & 330.8 \\
\hline & & {$[10.34]$} & {$[12.86]$} & {$[4.53]$} & [10.46] & {$[1.02]$} & {$[0.41]$} & {$[9.28]$} & [16.54] \\
\hline & T. cati B6 & 115.8 & 65.0 & 174.4 & 248.3 & 89.0 & 12.0 & 185.6 & 263.5 \\
\hline & & [5.79] & {$[3.25]$} & {$[8.72]$} & {$[12.42]$} & {$[4.45]$} & {$[0.60]$} & {$[9.28]$} & [13.18] \\
\hline & T. canis B6 & 75.1 & 44.1 & 57.4 & 51.4 & 62.0 & 59.8 & 56.1 & 40.6 \\
\hline & & {$[3.76]$} & {$[2.21]$} & {$[2.87]$} & {$[2.57]$} & {$[3.1]$} & {$[2.99]$} & {$[2.81]$} & [2.03] \\
\hline
\end{tabular}



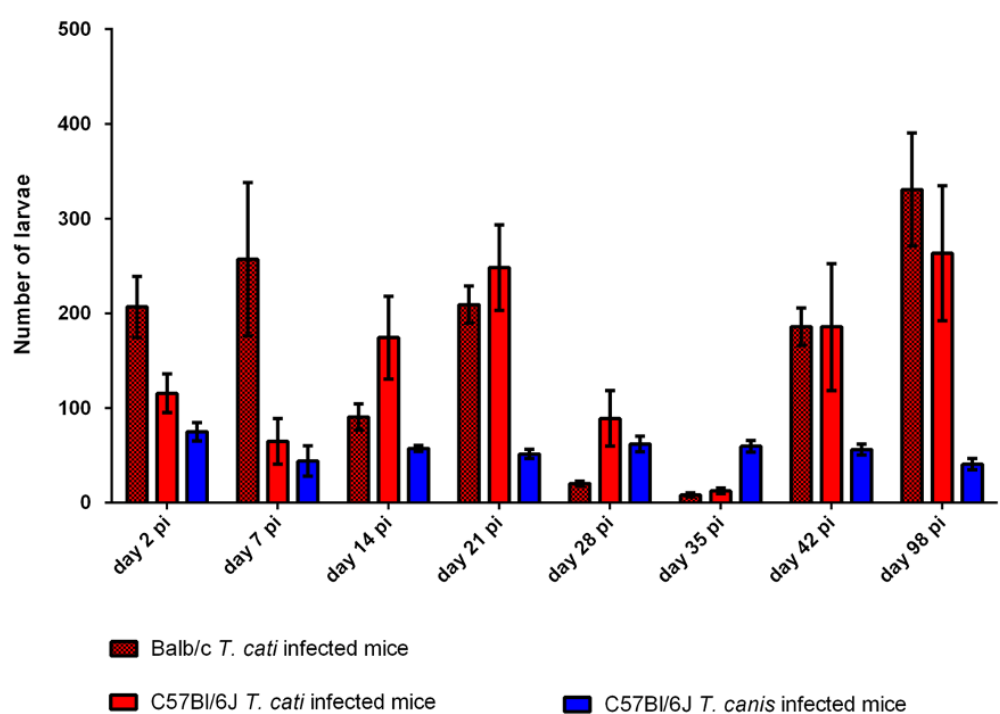

C57BI/6J T. canis infected mice

Figure 1 Total recovered larvae. Total mean number of T. cati and T. canis larvae recovered from experimentally infected Balb/c and B6 mice during different time points post infection. Error bars indicate standard errors of the mean (SEM).

infected mice. As in lungs, hemorrhages were reabsorbed starting at day 14 pi and only observed sporadically during the subsequent course of infection. Histopathology revealed changes in brain structure in all infection groups; however, changes in $T$. canis infected mice were more severe than those in T. cati infected mice. From day $14 \mathrm{pi}$ onwards, structural brain damage intensified in all infection groups, reaching a maximum of structural damage on the last study day 98 pi. It was apparent that structural damage was mainly observed in the cerebellum of $T$. cati infected mice whereas structural damage was also frequently observed in the cerebrum of $T$. canis infected mice. Histological changes included malacia (T. canis infected mice) with the presence of activated microglia and focal accumulation of gitter cells, which are phagocytic cells with the presence of myelin debris within the cytoplasm (myelinophages). Furthermore, occurrence of swollen axons (spheroids), indicative of axonal damage, was observed. Structural damage is shown exemplarily in Figure 6. The subjective impression was that $T$. canis infected mice started to exhibit slight balance problems starting around day $70 \mathrm{pi}$. Both T. canis and T. cati infected B6 mice seemed less aggressive day 98 pi when compared to earlier time points.

\section{Discussion}

The migrational route of $T$. canis larvae in the paratenic host, especially in mice, has been examined in various studies resulting in a strong affinity to the brain [10,33-35]. However, very few studies deal with the migrational behaviour of $T$. cati larvae in the paratenic host even though the zoonotic potential is not sufficiently evaluated and should therefore not be underestimated [11]. Direct comparison of studies dealing with either $T$. canis or $T$. cati is difficult and inaccurate as the course of infection is mouse strain dependent as well as depending on infection dose and day post infection $[18,28,30,31,36,37]$. To overcome this shortcoming, the present study provides a direct comparison of both roundworm species in mice with specific focus on the CNS in order to demonstrate possible brain damage caused by $T$. cati and $T$. canis larvae, respectively, during the acute and the chronic phase of neurotoxocarosis.

Table 2 Statistically significant differences of organ larval distribution between infection groups

\begin{tabular}{|c|c|c|c|c|c|c|c|c|}
\hline & \multicolumn{8}{|c|}{ Day pi } \\
\hline & 2 & 7 & 14 & 21 & 28 & 35 & 42 & 98 \\
\hline T. cati Balb/c vs. T. cati B6 & Lu & & & & & & & Cer, LH \\
\hline T. cati Balb/c vs. T. canis B6 & $\mathrm{Li}, \mathrm{Lu}, \mathrm{Mu}$ & $\mathrm{Br}, \mathrm{Mu}$ & $\begin{array}{c}\mathrm{Br}, \mathrm{Ceb}, \mathrm{LH}, \\
\mathrm{RH}, \mathrm{Mu}\end{array}$ & $\begin{array}{c}\text { Br, Ceb, LH, } \\
\text { RH, Li, Mu }\end{array}$ & $\begin{array}{c}\text { Br, Ceb, Cbell, } \\
\text { LH, RH, Mu }\end{array}$ & $\begin{array}{l}\text { Ceb, Cbell, } \\
\mathrm{LH}, \mathrm{RH}, \mathrm{Mu}\end{array}$ & $\begin{array}{c}\mathrm{Br}, \mathrm{Ceb}, \mathrm{LH}, \\
\mathrm{Lu}, \mathrm{Mu}\end{array}$ & $\begin{array}{l}\text { Br, Ceb, } \\
\text { Cbell, Mu }\end{array}$ \\
\hline T. cati B6 vs. T. canis B6 & $\mathrm{Li}, \mathrm{Lu}, \mathrm{Mu}$ & $\mathrm{Br}, \mathrm{Mu}$ & $\begin{array}{c}\mathrm{Br}, \mathrm{Ceb}, \mathrm{LH}, \\
\mathrm{RH}, \mathrm{Mu}\end{array}$ & $\begin{array}{c}\text { Br, Ceb, LH, } \\
\text { RH, Li, Mu }\end{array}$ & $\begin{array}{c}\text { Br, Ceb, Cbell, } \\
\text { LH, RH, Mu }\end{array}$ & $\begin{array}{l}\text { Ceb, Cbell, } \\
\text { LH, RH, Mu }\end{array}$ & $\begin{array}{l}\mathrm{Br}, \mathrm{Ceb}, \mathrm{LH}, \\
\mathrm{RH}, \mathrm{Lu}, \mathrm{Mu}\end{array}$ & $\begin{array}{l}\mathrm{Br}, \mathrm{Ceb}, \\
\mathrm{LH}, \mathrm{Mu}\end{array}$ \\
\hline
\end{tabular}


Table 3 Statistically significant differences of organ larval distribution within infection groups over course of infection

\begin{tabular}{|c|c|c|c|c|c|c|c|c|c|}
\hline \multirow[b]{2}{*}{ Day pi } & \multirow[b]{2}{*}{ Infection group } & \multicolumn{8}{|c|}{ Day pi } \\
\hline & & 2 & 7 & 14 & 21 & 28 & 35 & 42 & 98 \\
\hline \multirow[t]{3}{*}{2} & T. cati Balb/c & - & Ceb, Li, Lu, Mu & Ceb, Li, Lu, Mu & Ceb, Li, Lu, Mu & Ceb, Li, Lu, Mu & Ceb, Li, Lu, Mu & Ceb, Cbell, RH, Li, Lu, Mu & Cbell, LH, RH, Li, Lu, Mu \\
\hline & T. cati B6 & - & $\mathrm{Li}, \mathrm{Lu}, \mathrm{Mu}$ & $\mathrm{Li}, \mathrm{Lu}, \mathrm{Mu}$ & $\mathrm{Li}, \mathrm{Lu}, \mathrm{Mu}$ & $\mathrm{Li}, \mathrm{Lu}, \mathrm{Mu}$ & $\mathrm{Li}, \mathrm{Lu}, \mathrm{Mu}$ & $\mathrm{Li}, \mathrm{Lu}, \mathrm{Mu}$ & $\mathrm{Li}, \mathrm{Lu}, \mathrm{Mu}$ \\
\hline & T. canis B6 & - & $\mathrm{Br}, \mathrm{Ceb}, \mathrm{Li}, \mathrm{Mu}$ & $\mathrm{Br}, \mathrm{Li}, \mathrm{Mu}$ & $\mathrm{Br}, \mathrm{Li}, \mathrm{Mu}$ & $\mathrm{Li}, \mathrm{Mu}$ & $\mathrm{Br}$, Cbell, Li, Mu & $\mathrm{Br}$, Cbell, LH, Li, Mu & $\mathrm{Br}, \mathrm{Li}, \mathrm{Mu}$ \\
\hline \multirow[t]{3}{*}{7} & T. cati Balb/c & $\mathrm{Li}, \mathrm{Lu}, \mathrm{Mu}$ & - & & & & & Cbell, RH & Cbell, LH, RH \\
\hline & T. cati B6 & $\mathrm{Li}, \mathrm{Lu}, \mathrm{Mu}$ & - & $\mathrm{Lu}, \mathrm{Mu}$ & $\mathrm{Lu}, \mathrm{Mu}$ & $\mathrm{Lu}, \mathrm{Mu}$ & $\mathrm{Lu}, \mathrm{Mu}$ & $\mathrm{Lu}, \mathrm{Mu}$ & $\mathrm{Lu}, \mathrm{Mu}$ \\
\hline & T. canis B6 & $\mathrm{Br}, \mathrm{Ceb}, \mathrm{Li}, \mathrm{Mu}$ & - & $\mathrm{Mu}$ & $\mathrm{Mu}$ & $\mathrm{Br}, \mathrm{Mu}$ & & & \\
\hline \multirow[t]{3}{*}{14} & T. cati Balb/c & $\mathrm{Li}, \mathrm{Lu}, \mathrm{Mu}$ & & - & & & & Cbell, RH & Cbell, LH, RH \\
\hline & T. cati $\mathrm{B} 6$ & $\mathrm{Li}, \mathrm{Lu}, \mathrm{Mu}$ & $\mathrm{Lu}, \mathrm{Mu}$ & - & & & & & \\
\hline & T. canis B6 & $\mathrm{Br}, \mathrm{Li}, \mathrm{Mu}$ & $\mathrm{Mu}$ & - & & & & $\mathrm{Br}$ & \\
\hline \multirow[t]{3}{*}{21} & T. cati Balb/c & $\mathrm{Li}, \mathrm{Lu}, \mathrm{Mu}$ & & & - & & & Cbell, RH & Cbell, LH, RH \\
\hline & T. cati B6 & $\mathrm{Li}, \mathrm{Lu}, \mathrm{Mu}$ & $\mathrm{Lu}, \mathrm{Mu}$ & & - & & & & \\
\hline & T. canis B6 & $\mathrm{Br}, \mathrm{Li}, \mathrm{Mu}$ & $\mathrm{Mu}$ & & - & & & $\mathrm{Br}$, Cbell & \\
\hline \multirow[t]{3}{*}{28} & T. cati Balb/c & $\mathrm{Li}, \mathrm{Lu}, \mathrm{Mu}$ & & & & - & & Cbell, RH & Cbell, LH, RH \\
\hline & T. cati B6 & $\mathrm{Li}, \mathrm{Lu}, \mathrm{Mu}$ & $\mathrm{Lu}, \mathrm{Mu}$ & & & - & & & \\
\hline & T. canis B6 & $\mathrm{Li}, \mathrm{Mu}$ & $\mathrm{Br}, \mathrm{Mu}$ & & & - & $\mathrm{Br}$ & $\mathrm{Br}, \mathrm{Mu}$ & $\mathrm{Br}$ \\
\hline \multirow[t]{3}{*}{35} & T. cati Balb/c & $\mathrm{Li}, \mathrm{Lu}, \mathrm{Mu}$ & & & & & - & Cbell, RH & Cbell, LH, RH \\
\hline & T. cati B6 & $\mathrm{Li}, \mathrm{Lu}, \mathrm{Mu}$ & $\mathrm{Lu}, \mathrm{Mu}$ & & & & - & $\mathrm{Mu}$ & \\
\hline & T. canis B6 & $\mathrm{Br}$, Cbell, Li, Mu & & & & $\mathrm{Br}$, Cbell & - & & \\
\hline \multirow[t]{3}{*}{42} & T. cati Balb/c & Cbell, RH, Li, Lu, Mu & Cbell, RH & Cbell, RH & Cbell, RH & Cbell, RH & Cbell, RH & - & Cbell, LH \\
\hline & T. cati B6 & $\mathrm{Li}, \mathrm{Lu}, \mathrm{Mu}$ & $\mathrm{Lu}, \mathrm{Mu}$ & & & & & - & \\
\hline & T. canis B6 & $\mathrm{Br}$, Cbell, LH, Li, Mu & & $\mathrm{Br}$ & $\mathrm{Br}$, Cbell & $\mathrm{Br}$, Cbell, Mu & & - & \\
\hline \multirow[t]{3}{*}{98} & T. cati Balb/c & Cbell, LH, RH, Li, Lu, Mu & Cbell, LH, RH & Cbell, LH, RH & Cbell, LH, RH & Cbell, LH, RH & Cbell, LH, RH & Cbell & - \\
\hline & T. cati B6 & $\mathrm{Li}, \mathrm{Lu}, \mathrm{Mu}$ & $\mathrm{Lu}, \mathrm{Mu}$ & & & & & & - \\
\hline & T. canis B6 & $\mathrm{Br}, \mathrm{Li}, \mathrm{Mu}$ & & & & $\mathrm{Br}$ & & & - \\
\hline
\end{tabular}

Larval distribution in single organs within infection groups over the course of infection. Respective comparisons of days pi for each infection group are displayed. Significances were calculated using 2 -way ANOVA. $B r=$ total brain, $C e b=$ cerebrum, $C b e l l=$ cerebellum, $L H=$ left hemisphere, $R H=$ right hemisphere, $L i=$ liver, $L u=$ lungs, $M u=$ muscle. 
Table 4 P-values resulting from statistical comparison of larval distribution between organs within infection groups

\begin{tabular}{llcccccccc}
\hline & Infection group & \multicolumn{7}{c}{ Day pi } \\
\cline { 3 - 9 } & & $\mathbf{2}$ & $\mathbf{7}$ & $\mathbf{1 4}$ & $\mathbf{2 1}$ & $\mathbf{2 8}$ & $\mathbf{3 5}$ & $\mathbf{4 2}$ & $\mathbf{9 8}$ \\
\hline Brain vs. lungs & T. cati Balb/c & 0.0550 & 0.3699 & $0.0323^{*}$ & $0.0209^{*}$ & 0.3632 & 0.1782 & $<.0001^{*}$ & $0.0036^{*}$ \\
& T. cati B6 & $0.0008^{*}$ & 0.3945 & 0.0749 & 0.4868 & 0.2467 & 0.9724 & $0.0012^{*}$ & $0.0001^{*}$ \\
& T. canis B6 & $0.0434^{*}$ & $0.0179^{*}$ & $<.0001^{*}$ & $<.0001^{*}$ & $0.0107^{*}$ & $0.0015^{*}$ & $<.0001^{*}$ & $<.0001^{*}$ \\
Brain vs. liver & T. cati Balb/c & 0.3669 & 0.5658 & 0.1847 & 0.0891 & n.a. & 0.1782 & $0.0008^{*}$ & $0.0082^{*}$ \\
& T. cati B6 & $0.0001^{*}$ & 0.0520 & 0.7520 & $0.0039^{*}$ & 0.2179 & 0.3632 & $0.0344^{*}$ & $<.0001^{*}$ \\
Brain vs. muscle & T. canis B6 & $<.0001^{*}$ & $0.0271^{*}$ & $0.0007^{*}$ & $0.0004^{*}$ & $0.0211^{*}$ & $<.0001^{*}$ & $<.0001^{*}$ & $<.0001^{*}$ \\
& T. cati Balb/c & $0.009^{*}$ & $0.0333^{*}$ & $0.001^{*}$ & $<.0001^{*}$ & $<.0001^{*}$ & $0.0015^{*}$ & $0.0002^{*}$ & $0.0013^{*}$ \\
Cerebrum vs. cerebellum & T. cati B6 & $0.0002^{*}$ & $0.0020^{*}$ & $<.0001^{*}$ & $0.0002^{*}$ & $0.0002^{*}$ & $0.0005^{*}$ & $<.0001^{*}$ & $<.0001^{*}$ \\
& T. canis B6 & 0.2754 & 0.7050 & $0.0017^{*}$ & $0.0016^{*}$ & $0.0119^{*}$ & 0.5447 & 0.3359 & 0.7466 \\
& T. cati Balb/C & 0.4956 & $0.0264^{*}$ & $0.0355^{*}$ & 0.7864 & 0.3632 & n.a. & $0.0403^{*}$ & 0.0903 \\
& T. cati B6 & 0.4063 & $0.0454^{*}$ & 0.0705 & 0.1195 & 0.6109 & 0.3632 & 0.8607 & $0.0033^{*}$ \\
Left vs. right hemisphere & T. canis B6 & 1.0000 & $0.0154^{*}$ & 0.2918 & $0.0040^{*}$ & $0.0003^{*}$ & $0.0465^{*}$ & $0.0441^{*}$ & $0.0136^{*}$ \\
& T. cati Balb/c & 0.3610 & 0.2636 & 0.5224 & 0.9766 & 0.3632 & 1.0000 & 0.1424 & 0.7825 \\
& T. cati B6 & 0.4063 & 0.9585 & 0.1787 & 0.9075 & 0.6109 & n.a. & 0.2565 & 0.4993 \\
& T. canis B6 & 0.1747 & 0.7284 & 0.1622 & 0.3854 & 0.6336 & 0.7935 & 0.4490 & 0.9738 \\
\hline
\end{tabular}

Comparison of larval distribution between single organs at a given day pi for each infection group. P-values were calculated using LSD post hoc tests.

*: statistically significant ( $\mathrm{p} \leq 0.05)$.

n.a.: not applicable (too low larvae numbers for reliable comparison).

Concerning macroscopical changes in the brain, hemorrhagic lesions observed on day $7 \mathrm{pi}$ in the infection groups were also confirmed by histology. It has been proposed that those lesions are caused by larval penetration of arteries in the cortex resulting in injury and consequent bleeding [38]. Lesions within the first week of infection with resulting reabsorption had also been observed in $T$. cati and $T$. canis infected mice by other authors $[15,38,39]$ but could not be observed in a study by Dubey [17]. Histology revealed the presence of malacia in T. canis infected mice. Activated microglia and gitter cells, possibly indicating demyelination, as well as spheroid formation as a hallmark of axonal damage were observed in all infection groups. These changes are considered a response of the brain to the migrating or arrested larvae.

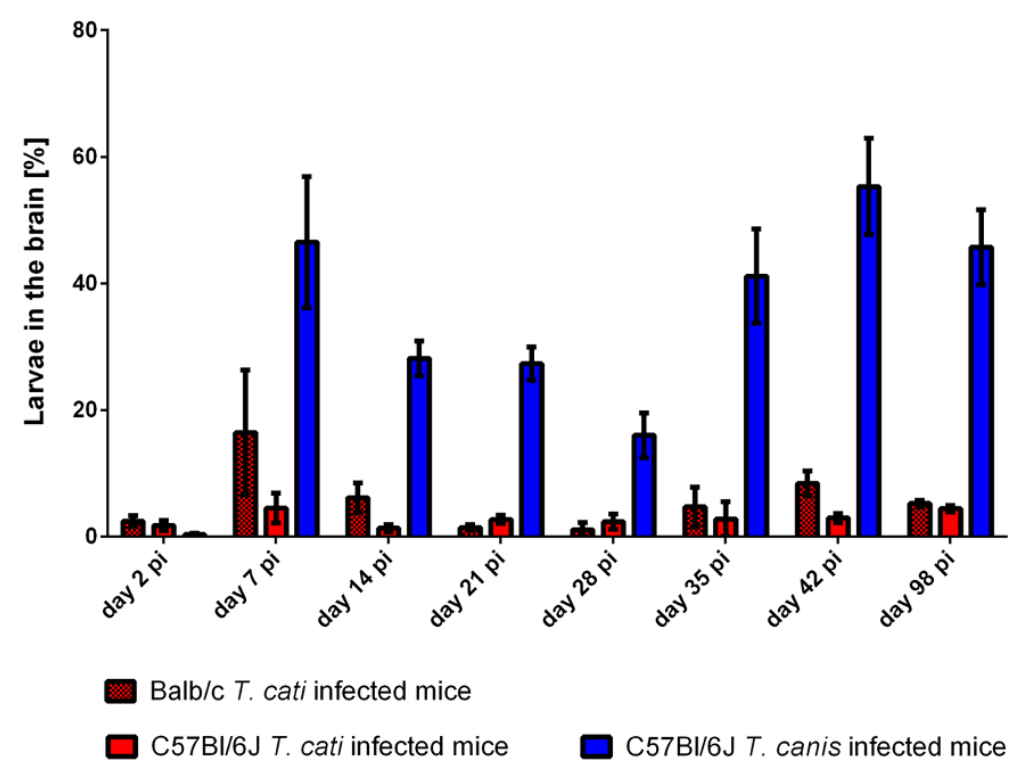

Figure 2 Larval recovery rates in the brain. Recovery rates in [\%] of T. cati and T. canis larvae from the brain based on the total number of recovered larvae in experimentally infected mice during different time points pi. Error bars indicate standard errors of the mean (SEM). 


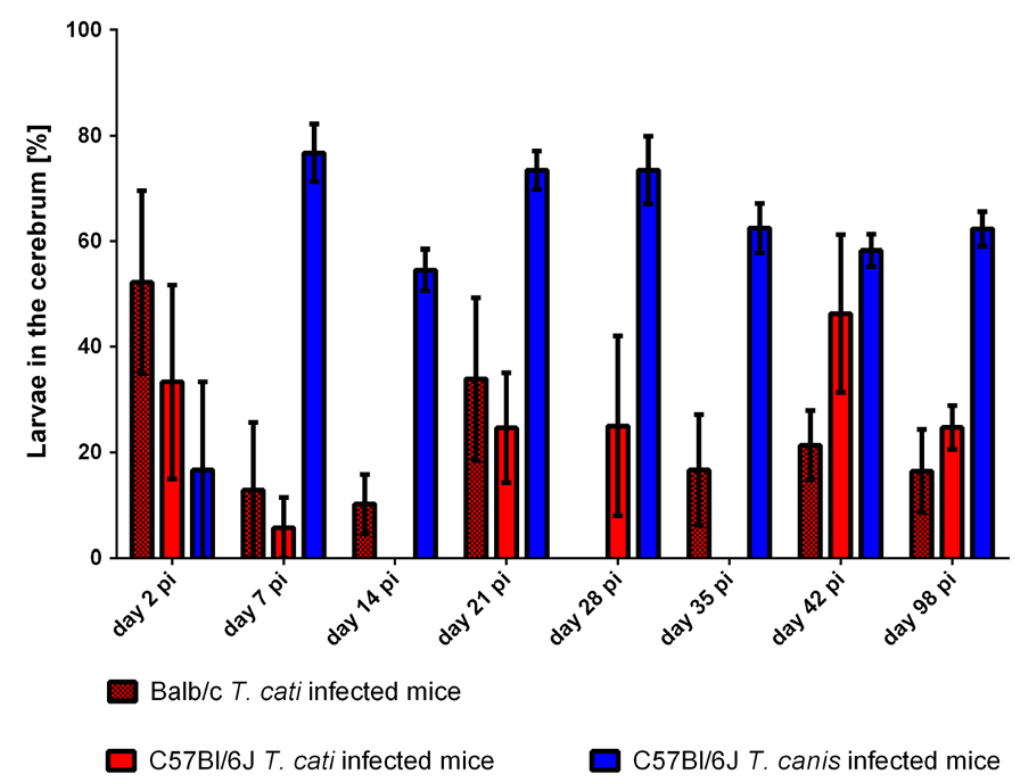

Figure 3 Larval recovery rates in the cerebrum. Recovery rates in [\%] of T. cati and T. canis larvae from the cerebrum based on the total larval number found in the brain of experimentally infected mice during different time points pi. Error bars indicate standard errors of the mean (SEM).

The general data obtained show that there are significant differences between infection groups during the acute phase of infection. The higher larval numbers of T. canis in the liver in contrast to significantly higher numbers of T. cati larvae in the lungs on day 2 pi provides evidence that $T$. canis larvae migrate at a slower pace than $T$. cati larvae. In a previous study, the migrational route was divided into two phases namely the hepato-pulmonary phase and the visceral phase. During the hepato-pulmonary phase, larvae penetrate the intestinal wall after hatching and migrate to the liver. From there on, migration is continued via systemic circulation through which larvae reach the lungs [40]. The slower migration pace of $T$. canis has been observed in mice before [16], however, other studies indicated a slower migration route of $T$. cati in the Mongolian gerbil as paratenic host [33].

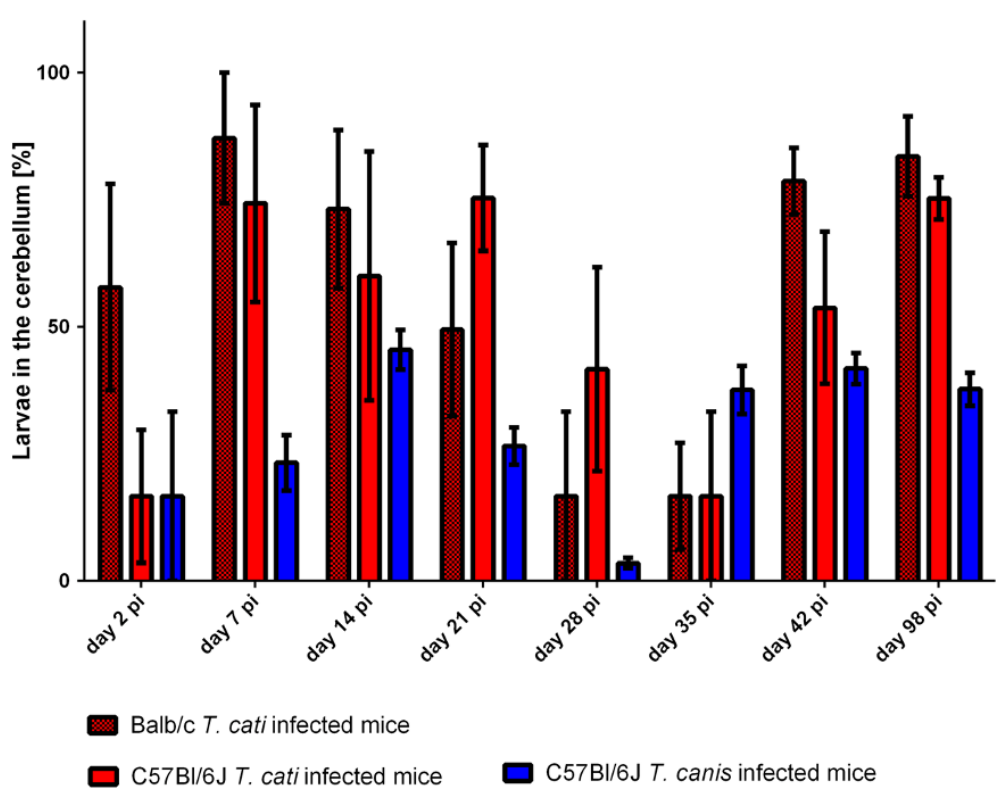

Figure 4 Larval recovery rates in the cerebellum. Recovery rates in [\%] of T. cati and T. canis larvae from the cerebellum based on the total larval number found in the brain of experimentally infected mice during different time points pi. Error bars indicate standard errors of the mean (SEM). 
Table 5 Larval distribution and recovery rates in CNS organs

\begin{tabular}{|c|c|c|c|c|c|c|c|c|c|c|c|c|c|c|c|c|c|c|c|c|c|c|c|c|c|}
\hline \multirow[t]{3}{*}{ CNS organ } & \multirow[t]{3}{*}{ Infection group } & \multicolumn{24}{|c|}{ Day pi } \\
\hline & & \multicolumn{3}{|c|}{2} & \multicolumn{3}{|c|}{7} & \multicolumn{3}{|c|}{14} & \multicolumn{3}{|c|}{21} & \multicolumn{3}{|c|}{28} & \multicolumn{3}{|c|}{35} & \multicolumn{3}{|c|}{42} & \multicolumn{3}{|c|}{98} \\
\hline & & $\underline{\mathrm{LH}}$ & RH & $\mathbf{T}$ & LH & RH & $\mathbf{T}$ & LH & RH & $\mathbf{T}$ & LH & RH & $\mathrm{T}$ & LH & RH & $\mathrm{T}$ & LH & RH & $\mathbf{T}$ & LH & RH & $\mathrm{T}$ & LH & RH & $\mathbf{T}$ \\
\hline \multirow[t]{3}{*}{ Cerebrum } & T. cati Balb/c & 1.4 & 1.2 & 2.6 & 1.8 & 0.0 & 1.8 & 0.5 & 0.3 & 0.8 & 0.5 & 0.5 & 1.0 & 0.0 & 0.0 & 0.0 & 0.2 & 0.2 & 0.3 & 0.8 & 2.3 & 3.2 & 1.7 & 2.0 & 3.7 \\
\hline & T. cati B6 & 1.3 & 0.5 & 1.8 & 0.4 & 0.0 & 0.4 & 0.0 & 0.0 & 0.0 & 1.2 & 0.0 & 1.2 & 0.3 & 0.0 & 0.3 & 0.0 & 0.0 & 0.0 & 0.7 & 0.8 & 1.5 & 1.5 & 0.5 & 2.0 \\
\hline & T. canis B6 & 0.0 & 0.2 & 0.2 & 12.0 & 13.6 & 25.6 & 4.2 & 4.8 & 9.0 & 6.3 & 4.7 & 11.0 & 4.8 & 3.2 & 8.0 & 10.2 & 6.8 & 17.2 & 10.8 & 9.5 & 20.3 & 5.8 & 7.3 & 13.2 \\
\hline \multirow[t]{3}{*}{ Cerebellum } & T. cati Balb/c & 0.2 & 1.4 & 1.6 & 1.8 & 1.2 & 3.0 & 2.7 & 2.3 & 5.0 & 1.2 & 1.2 & 2.3 & 0.2 & 0.0 & 0.2 & 0.2 & 0.2 & 0.3 & 3.7 & 8.3 & 12.0 & 10.3 & 7.7 & 18.0 \\
\hline & T. cati B6 & 0.2 & 0.8 & 1.0 & 0.8 & 1.2 & 2.0 & 2.2 & 1.2 & 3.4 & 1.8 & 3.0 & 4.8 & 0.3 & 0.3 & 0.5 & 0.2 & 0.2 & 0.3 & 3.3 & 2.0 & 5.3 & 2.8 & 4.2 & 7.0 \\
\hline & T. canis B6 & 0.0 & 0.2 & 0.2 & 3.0 & 3.6 & 6.6 & 2.5 & 5.2 & 7.7 & 2.0 & 2.2 & 4.2 & 1.5 & 2.0 & 3.5 & 6.0 & 4.8 & 10.7 & 7.5 & 6.8 & 14.3 & 4.7 & 3.5 & 8.2 \\
\hline \multirow[t]{3}{*}{ Total brain } & T. cati Balb/c & 1.6 & 2.6 & 4.2 & 3.6 & 1.2 & 4.8 & 3.2 & 2.7 & 5.8 & 1.7 & 1.7 & 3.3 & 0.2 & 0.0 & 0.2 & 0.3 & 0.3 & 0.7 & 4.5 & 10.7 & 15.2 & 12.0 & 9.7 & 21.7 \\
\hline & T. cati B6 & 1.5 & 1.3 & 2.8 & 1.2 & 1.2 & 2.4 & 2.2 & 1.2 & 3.4 & 3.0 & 3.0 & 6.0 & 0.5 & 0.3 & 0.8 & 0.2 & 0.2 & 0.3 & 4.0 & 2.8 & 6.8 & 4.3 & 4.7 & 9.0 \\
\hline & T. canis B6 & 0.0 & 0.3 & 0.3 & 15.0 & 17.2 & 32.2 & 6.7 & 10.0 & 16.7 & 8.3 & 6.8 & 15.2 & 6.3 & 5.2 & 11.5 & 16.2 & 11.2 & 27.8 & 18.3 & 16.3 & 34.7 & 10.5 & 10.8 & 21.3 \\
\hline \multirow[t]{6}{*}{ Spinal cord } & \multirow{2}{*}{ T. cati Balb/c } & \multicolumn{3}{|c|}{1.0} & \multicolumn{3}{|c|}{0.7} & \multicolumn{3}{|c|}{0.3} & \multicolumn{3}{|c|}{1.0} & \multicolumn{3}{|c|}{0.0} & \multicolumn{3}{|c|}{0.0} & \multicolumn{3}{|c|}{0.0} & \multicolumn{3}{|c|}{0.6} \\
\hline & & & [0.39] & & & {$[0.32]$} & & & {$[0.25]$} & & & {$[0.50]$} & & & [0.00] & & & {$[0.00]$} & & & {$[0.00]$} & & & {$[0.19]$} & \\
\hline & $T$ sati BG & & 0.9 & & & 1.3 & & & 0.4 & & & 1.1 & & & 0.3 & & & 0.0 & & & 0.0 & & & 0.4 & \\
\hline & 1. call do & & [0.48] & & & [2.02] & & & [0.19] & & & {$[0.45]$} & & & [0.13] & & & {$[0.00]$} & & & {$[0.00]$} & & & [0.30] & \\
\hline & I sanis B6 & & 0.0 & & & 1.3 & & & 0.4 & & & 0.9 & & & 0.9 & & & 0.6 & & & 1.1 & & & 1.5 & \\
\hline & 1. contis do & & {$[0.00]$} & & & [3.46] & & & [0.64] & & & {$[1.85]$} & & & [1.77] & & & {$[0.09]$} & & & [2.03] & & & [3.31] & \\
\hline Eyes & $T$ cati Ralb/a & & 1.6 & & & 0.7 & & & 0.0 & & & 0.0 & & & 0.0 & & & 0.0 & & & 0.1 & & & 0.6 & \\
\hline & 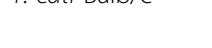 & & {$[1.01]$} & & & {$[0.63]$} & & & {$[0.00]$} & & & {$[0.00]$} & & & {$[0.00]$} & & & {$[0.00]$} & & & {$[0.07]$} & & & {$[0.31]$} & \\
\hline & T sati BG & & 0.3 & & & 0.1 & & & 0.0 & & & 0.3 & & & 0.0 & & & 0.0 & & & 0.0 & & & 0.0 & \\
\hline & 1. cati do & & [0.32] & & & {$[0.65]$} & & & {$[0.00]$} & & & {$[0.15]$} & & & {$[0.00]$} & & & {$[0.00]$} & & & {$[0.00]$} & & & {$[0.00]$} & \\
\hline & $T$ sanis B6 & & 0.0 & & & 0.4 & & & 0.3 & & & 0.1 & & & 0.1 & & & 0.3 & & & 0.5 & & & 0.1 & \\
\hline & 1. Camis Do & & {$[0.00]$} & & & [2.84] & & & {$[0.4]$} & & & {$[0.42]$} & & & {$[0.12]$} & & & {$[0.32]$} & & & {$[0.70]$} & & & {$[0.23]$} & \\
\hline
\end{tabular}

Mean larval numbers as well as recovery rates in [\%] for examined eyes, spinal cord and brain (brain recovery rates are pictured in Figures 2 and 3). Brain larval numbers are divided into total brain as well as cerebrum and cerebellum ( $L H=$ left hemisphere; $R H=$ right hemisphere; $T=$ total organ). Total numbers may slightly differ as calculations are based on individual mean numbers and therefore, slight discrepancies occasionally occur when totaling values of different brain parts. 


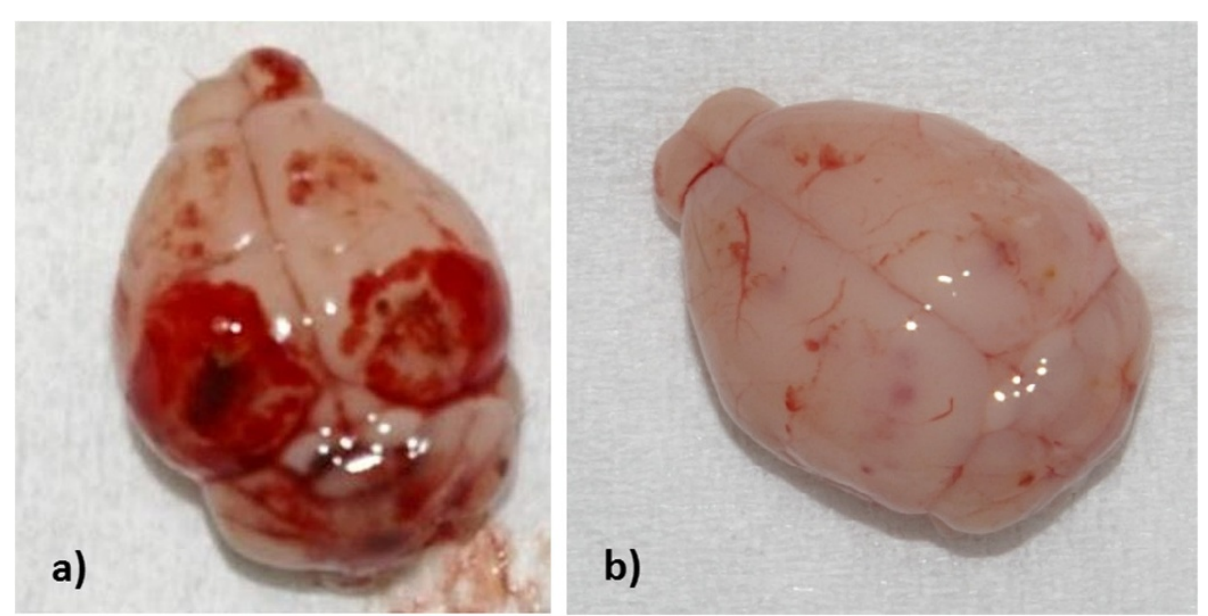

Figure 5 Macroscopical changes in the brain. Hemorrhagic lesions in brains of a) B6 T. canis infected mice and b) B6 T. cati infected mice on day 7 pi.

The $T$. cati course of infection was unexpected as total recovery rates severely dropped on day 14 pi as well as day 28 and 35 pi in Balb/c T. cati infected mice and on day 28 and 35 pi in B6 T. cati infected mice. In previous studies, inconsistent recovery rates (between 25\%-65\%) have been observed; however, those studies solely investigated day 1-28 pi and not the later time points post infection [17]. Initially, it could be assumed that larvae are eliminated from the host's tissue, however, the increase of recovery rates starting day 42 pi leads to the conclusion that an active migration and shift of location occurs between day 28 and 35 pi as larval numbers in liver and lungs repeatedly increase starting day $35 \mathrm{pi}$ as well as larval numbers in the brain starting day 42 pi.
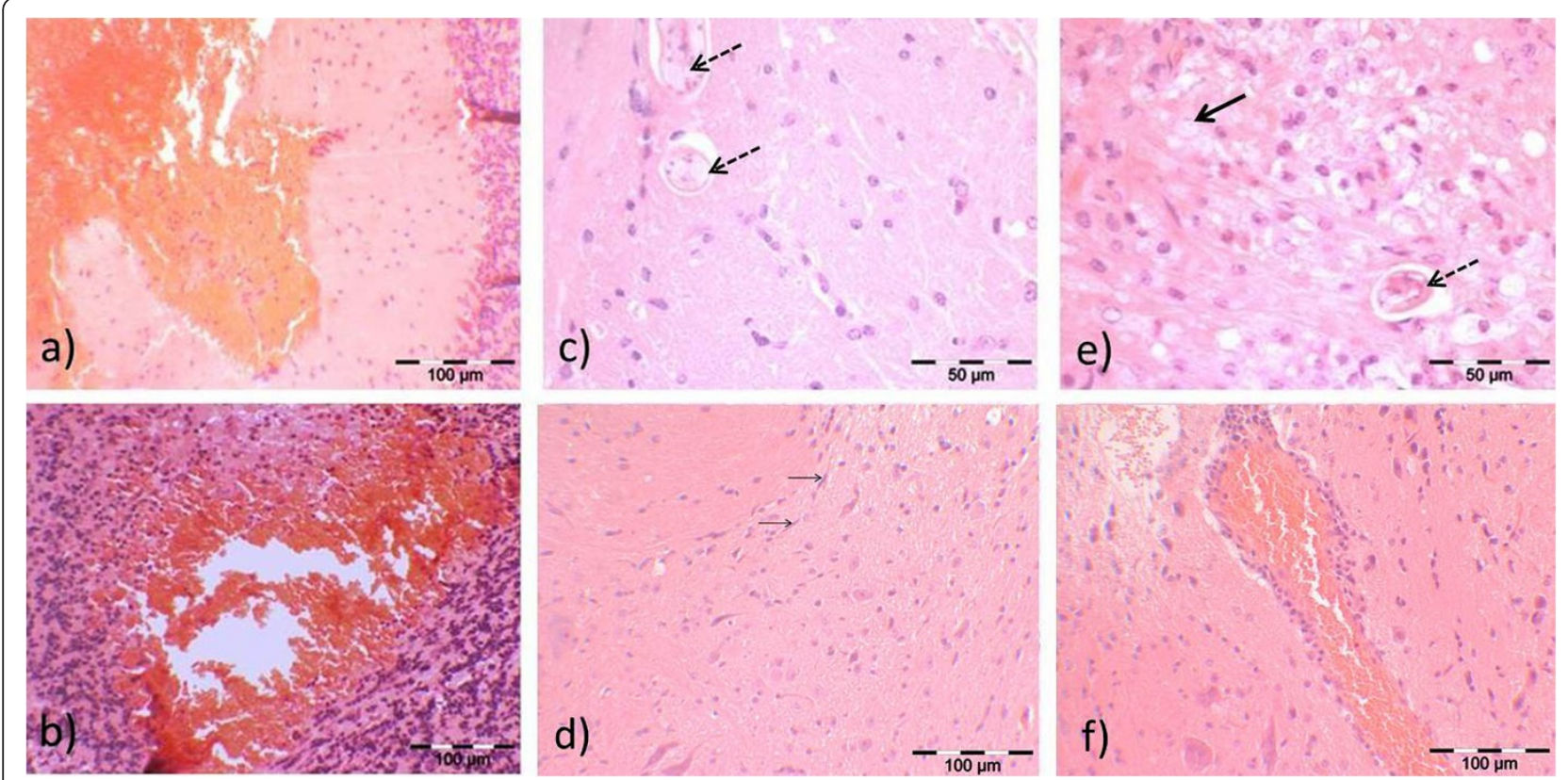

Figure 6 Histopathological changes in brain structure. Structural damage observed in brains of experimentally infected B6 mice during the course of infection. a) T. canis and b) T. cati infected mice on day 7 pi. Visible hemorrhages confirm macroscopic observations (cf. Figure 5). c) T. canis and d) T. cati infected mice day 42 pi. In c) T. canis larvae (dashed arrows) are detected in the neuroparenchyma. In d), mild microgliosis is observed. Thin arrows show activated microglia. e) T. canis and f) T. cati infected mice day 98 pi. Structural damage has intensified since day 42 pi. Generally, in T. canis infected mice structural damage was evaluated as more severe than in T. cati infected mice. In e), arrested or migrating $T$. canis larvae (dashed arrows) can be found. Malacia with demyelination and accumulation of gitter cells (thick arrows) is frequently observed. In f), perivascular lymphocytosis is visualized. 
Nevertheless, the tissue-residency and migrational route of larvae during these time points remains unclear. Overall, recovery rates of $T$. cati larvae were up to $16 \%$, which is comparable to about $15.9 \%$ on average in gerbils and higher than about $2.0 \%$ found in rats [41]. Recovery rates of up to $65.7 \%$ of the total infection dose have been determined previously, however, mice received $1000 \mathrm{~T}$. cati eggs, i.e. only half of the infection dose of the present study and only 2 mice were investigated per time point post infection leading to a presumably shifted average [15].

By contrast to previous studies on T. canis migration in the paratenic host which revealed recovery rates of up to $43.4 \%$ in B6 mice after infection with 1000 infective T. canis larvae $[18,30]$, comparably low T. canis recovery rates of only up to $3.75 \%$ were found in the present study. Mechanisms such as trapping of $T$. canis larvae, especially in $\mathrm{B} 6$ mice, in the liver had been hypothesized before [42,43]. Possibly, the high amount of infective eggs led to a more sufficient host response and larvae were prevented from entry into the systemic circulation and were simultaneously eliminated. Another explanation is insufficient hatching of eggs and subsequent excretion with the feces [16,17].

Even though low recovery rates were obtained in $T$. canis infected mice, the previously demonstrated strong affinity to the CNS was confirmed in this study. Even though occasionally more than $50 \%$ of recovered larvae were detected in muscle tissue, larval numbers may be extrapolated when taking the mass of brain and muscle into consideration resulting in an assumed affinity to brain tissue, which may also be a result of better larval adjustment to brain tissue as opposed to other body tissues. Contrarily, significantly more T. cati larvae were found in muscle tissue than in brain tissue during all time points post infection. It has been hypothesized that the pronounced affinity of $T$. canis to the CNS is explained by the size difference when compared to $T$. cati larvae. Consequently, smaller $T$. cati larvae are able to leave arteries more easily, whereas $T$. canis larvae may finally arrest in the brain, as they are not likely to leave the circulatory system $[38,42]$. However, $T$. cati larvae were recovered in brains of both mouse strains whereas other studies indicated that migration of $T$. cati to the brain occurs rarely or not at all $[9,17,18]$. The increase of $T$. cati larvae in the brain during later time points post infection was also observed previously, when larval numbers increased starting at day 70 pi [41] as opposed to the current study where larval numbers in the brain start increasing at day 42 pi.

Looking at the left and right hemisphere, significant differences were detected between, but not within the infection groups. The significance of this observation needs to be further investigated as no data concerning left and right hemispheres are available. Additionally, structural damage appears to be consistently distributed in both hemispheres.

The frequent occurrence of $T$. cati larvae in the cerebellum as opposed to T. canis larvae in the cerebrum is rather surprising as previous studies demonstrated $T$. canis and T. cati larvae being predominantly present in the cerebellum $[9,10,33,38]$. Eventually, different paratenic hosts could lead to the shift of distribution in the brain. Additionally, due to size difference, the cerebrum is supplied by a greater amount of blood compared to the cerebrum and therefore more $T$. canis larvae may arrest in the small cerebrum arteries. Nevertheless, data obtained correlate well with histopathology as several changes were found in cerebra of $T$. canis infected mice, however, most structural damage in $T$. cati infected mice was observed in the cerebellum. As the cerebellum controls complex motor functions, the predominant occurrence of $T$. cati larvae in the cerebellum may be explained by possible clinical consequences like an increase of immobility and behavioral alterations, e.g. spending more time in open areas. Consequently, paratenic hosts are an easier prey for final hosts of Toxocara spp. [26,29,44,45].

Ocular and spinal cord involvement was demonstrated for all infection groups in the present study. Contrarily, previous studies solely demonstrated $T$. canis but not $T$. cati larvae in eyes of infected mice $[9,16]$. As only very low numbers of larvae were recovered from the eyes, it is apparent that mice are not a suitable model for $T$. canis or T. cati induced ocular larva migrans. However, it may not be excluded that OLM and possibly resulting symptoms may occur after infection with either species. Spinal cord involvement has been demonstrated previously in $T$. canis infected mice $[9,39]$ as well as rats, gerbils and hamsters [10], but information about spinal cord involvement in $T$. cati infected mice has not been available so far.

Even though lower percentages of $T$. cati larvae accumulate in the brain, intermittently, comparable numeric values of larvae to $T$. canis were found. Therefore, the risk of neurotoxocarosis caused by $T$. cati should not be underestimated. However, it has also been demonstrated in $T$. canis infected animals that the observed damages in brain structure do not necessarily correspond to the number of larvae present and that a high larval burden does not correlate with the severity of symptoms. Therefore, adverse immune mechanisms are hypothesized to be responsible for the pathology and not the migrating larvae [30]. Additionally, effects of larvae are assumed to be indirect as observed lesions were found to exist separately from the migrating larvae [33]. The low recovery rate of $T$. cati larvae may suggest mild or no brain damage, however, the host's immune mechanisms may still result in distinct pathology - as it was obvious by histopathological examination of the brains (cf. Figure 6). 
The infection risk for humans with $T$. cati has to be considered at least as high as infection risk with $T$. canis as prevalences for example in Europe, range from $3.5 \%-34 \%$ in dogs and from $8 \%-76 \%$ in cats [46-49] whereby cats are more likely to contaminate places like playgrounds, gardens, etc. due to their defecation habits. The resulting environmental contamination increases the risk of infection for humans. Additionally, consuming undercooked or raw meat serves as a transmission route, as several studies have demonstrated the persistence of $T$. canis and $T$. cati larvae in a number of paratenic hosts, which are sources of food for humans [50-53]. Furthermore, viable larvae of $T$. canis as well as $T$. cati have been found in meat even after prolonged periods of freezing $[7,8,54,55]$.

\section{Conclusions}

Even though lower numbers of $T$. cati than T. canis larvae were recovered from the brain, the zoonotic potential of $T$. cati should not be underestimated as indirect effects of the larvae may cause distinct pathology. The assumption that most human cases of neurotoxocarosis are caused by T. canis should be revised as it was shown, that even present in small numbers, structural damage in $T$. cati infected brains is found. Chronic symptoms after day $98 \mathrm{pi}$ - the last day of the present study - remain to be determined as larval numbers increase starting day 42 pi throughout day 98 pi. Further molecular studies analyzing the host's response to migrating or persisting larvae in the brain could provide a solid foundation for further investigations on neurotoxocarosis and evaluation of the disease. The occurrence of T. cati induced neurotoxocarosis and persistence of infective larvae in meat, even under severe conditions, highlights the need to characterize the course of infection to extrapolate it to human neurotoxocarosis as it may not be as rare as previously thought.

\section{Competing interests}

The authors declare that they have no competing interests.

\section{Authors' contributions}

EJ carried out animal experiments and laboratory work, analysed data and drafted the manuscript. AB analysed histopathological brain sections. CS designed and coordinated the study. TS designed and partially coordinated the study (TS deceased before concluding experiments). EJ, AB and CS interpreted results and read and approved the final manuscript.

\section{Acknowledgements}

The authors thank B. Buck for excellent technical assistance.

\section{Author details}

${ }^{1}$ Institute for Parasitology, University of Veterinary Medicine Hannover, Buenteweg 17, 30559 Hannover, Germany. ${ }^{2}$ Department of Pathology, University of Veterinary Medicine Hannover, Buenteweg 17, 30559 Hannover, Germany.

Received: 7 March 2014 Accepted: 14 April 2014

Published: 22 April 2014

\section{References}

1. Glickman LT, Schantz PM: Epidemiology and pathogenesis of zoonotic toxocariasis. Epidemiol Rev 1981, 3:230-250.

2. Brunaska M, Dubinsky P, Reiterova K: Toxocara canis: ultrastructural aspects of larval moulting in the maturing eggs. Int J Parasitol 1995, 25:683-690.

3. Overgaauw PA: Aspects of Toxocara epidemiology: toxocarosis in dogs and cats. Crit Rev Microbiol 1997, 23:23-251.

4. Sprent JF: On the migratory behavior of the larvae of various Ascaris species in white mice. I. Distribution of larvae in tissues. J Infect Dis 1952, 90:165-176.

5. Beaver PC: The nature of visceral larva migrans. J Parasitol 1969, 55:3-12.

6. Maruyama S, Nino T, Yamamoto K, Katsube Y: Parasitism of Toxocara canis larvae in chickens inoculated with the ascarid eggs. J Vet Med Sci 1994, 56:139-141.

7. Oryan A, Sadjjadi SM, Azizi S: Longevity of Toxocara cati larvae and pathology in tissues of experimentally infected chickens. Korean $J$ Parasitol 2010, 48:79-80.

8. Taira K, Saitoh Y, Kapel CM: Toxocara cati larvae persist and retain high infectivity in muscles of experimentally infected chickens. Vet Parasitol 2011, 180:287-291.

9. Burren $\mathrm{CH}$ : The distribution of Toxocara larvae in the central nervous system of the mouse. Trans R Soc Trop Med Hyg 1971, 65:450-453.

10. Burren $\mathrm{CH}$ : The distribution of Toxocara canis larvae in the central nervous system of rodents. Trans R Soc Trop Med Hyg 1972, 66:937-942.

11. Fisher M: Toxocara cati: an underestimated zoonotic agent. Trends Parasitol 2003, 19:167-170.

12. Skerrett $\mathrm{H}, \mathrm{Holland} \mathrm{CV}$ : Variation in the larval recovery of Toxocara canis from the murine brain: implications for behavioural studies. $J$ Helmintho 1997, 71:253-255

13. Othman AA, Abdel-Aleem GA, Saied EM, Mayah WW, Elatrash AM: Biochemical and immunopathological changes in experimental neurotoxocariasis. Mol Biochem Parasitol 2010, 172:1-8.

14. Liao CW, Fan CK, Kao TC, Ji DD, Su KE, Lin YH, Cho WL: Brain injuryassociated biomarkers of TGF-beta1, S100B, GFAP, NF-L, tTG, AbetaPP, and tau were concomitantly enhanced and the UPS was impaired during acute brain injury caused by Toxocara canis in mice. BMC Infect Dis 2008, 8:84

15. Cardillo N, Rosa A, Ribicich M, Lopez C, Sommerfelt I: Experimental infection with Toxocara cati in BALB/c mice, migratory behaviour and pathological changes. Zoonoses Public Health 2009, 56:198-205.

16. Prokopic J, Figallova V: Migration of some roundworm species in experimentally infected white mice. Folia Parasitol (Praha) 1982, 29:309-313.

17. Dubey JP: Migration of Toxocara cati larvae in mice. Trop Geogr Med 1968, 20:172-176.

18. Havasiova-Reiterova $K$, Tomasovicova O, Dubinsky P: Effect of various doses of infective Toxocara canis and Toxocara cati eggs on the humoral response and distribution of larvae in mice. Parasitol Res 1995, 81:13-17.

19. Hill IR, Denham DA, Scholtz CL: Toxocara canis larvae in the brain of a British child. Trans R Soc Trop Med Hyg 1985, 79:351-354.

20. Finsterer J, Auer H: Neurotoxocarosis. Rev Inst Med Trop Sao Paulo 2007, 49:279-287

21. Moreira-Silva SF, Rodrigues MG, Pimenta $\lrcorner$, Gomes CP, Freire LH, Pereira FE: Toxocariasis of the central nervous system: with report of two cases. Rev Soc Bras Med Trop 2004, 37:169-174.

22. Scheid R, Tina Jentzsch R, Schroeter ML: Cognitive dysfunction, urinary retention, and a lesion in the thalamus-beware of possible toxocariasis of the central nervous system. Clin Neurol Neurosurg 2008, 110:1054-1057.

23. Eberhardt $O$, Bialek R, Nagele T, Dichgans J: Eosinophilic meningomyelitis in toxocariasis: case report and review of the literature. Clin Neurol Neurosurg 2005, 107:432-438.

24. Walsh M, Haseeb MA: Reduced cognitive function in children with toxocariasis in a nationally representative sample of the United States. Int J Parasitol 2012, 42:1159-1163.

25. Fukae J, Kawanabe T, Akao N, Kado M, Tokoro M, Yokoyama K, Hattori N: Longitudinal myelitis caused by visceral larva migrans associated with Toxocara cati infection: Case report. Clin Neurol Neurosurg 2012, 114:1091-1094.

26. Holland CV, Cox DM: Toxocara in the mouse: a model for parasite-altered host behaviour? J Helminthol 2001, 75:125-135. 
27. Cox DM, Holland CV: Influence of mouse strain, infective dose and larval burden in the brain on activity in Toxocara-infected mice. $J$ Helminthol 2001, 75:23-32.

28. Cox DM, Holland CV: The relationship between numbers of larvae recovered from the brain of Toxocara canis-infected mice and social behaviour and anxiety in the host. Parasitology 1998, 116:579-594.

29. Hamilton CM, Stafford P, Pinelli E, Holland CV: A murine model for cerebral toxocariasis: characterization of host susceptibility and behaviour. Parasitology 2006, 132:791-801.

30. Epe C, Sabel T, Schnieder T, Stoye M: The behavior and pathogenicity of Toxocara canis larvae in mice of different strains. Parasitol Res 1994, 80:691-695.

31. Good B, Holland CV, Stafford P: The influence of inoculum size and time post-infection on the number and position of Toxocara canis larvae recovered from the brains of outbred CD1 mice. J Helminthol 2001, 75:175-181.

32. The Mouse Brain Library. http://www.mbl.org/mbl_main/atlas.html.

33. Akao N, Tomoda M, Hayashi E, Suzuki R, Shimizu-Suganuma M, Shichinohe K, Fujita K: Cerebellar ataxia due to Toxocara infection in Mongolian gerbils, Meriones unguiculatus. Vet Parasitol 2003, 113:229-237.

34. Glickman LT, Summers BA: Experimental Toxocara canis infection in cynomolgus macaques (Macaca fascicularis). Am J Vet Res 1983, 44:2347-2354

35. Alba-Hurtado F, Tortora PJ, Tsutsumi V, Ortega-Pierres MG: Histopathological investigation of experimental ocular toxocariasis in gerbils. Int J Parasitol 2000, 30:143-147.

36. Kayes SG, Omholt PE, Grieve RB: Immune responses of CBA/J mice to graded infections with Toxocara canis. Infect Immun 1985, 48:697-703.

37. Ollero MD, Fenoy S, Cuellar C, Guillen JL, Del Aguila C: Experimental toxocariosis in $\mathrm{BALB} / \mathrm{c}$ mice: effect of the inoculation dose on brain and eye involvement. Acta Trop 2008, 105:124-130.

38. Bisseru B: Studies on the liver, lung, brain and blood of experimental animals infected with Toxocara canis. J Helminthol 1969, 43:267-272.

39. Olson $\sqcup$, Petteway MB: Invasion of the spinal cord of mice by Toxocara canis. J Parasitol 1972, 58:413-414.

40. Abo-Shehada MN, Herbert IV: The migration of larval Toxocara canis in mice. II. Post-intestinal migration in primary infections. Vet Parasitol 1984 , 17:75-83.

41. Zibaei M, Sadjjadi SM, Uga S: Experimental Toxocara cati infection in gerbils and rats. Korean J Parasitol 2010, 48:331-333.

42. Dunsmore JD, Thompson RC, Bates IA: The accumulation of Toxocara canis larvae in the brains of mice. Int J Parasitol 1983, 13:517-521.

43. Parsons JC, Grieve RB: Effect of egg dosage and host genotype on liver trapping in murine larval toxocariasis. J Parasitol 1990, 76:53-58.

44. Chieffi PP, Aquino RT, Pasqualotti MA, Ribeiro MC, Nasello AG: Behavioral changes in Rattus norvegicus experimentally infected by Toxocara canis larvae. Rev Inst Med Trop Sao Paulo 2010, 52:243-246.

45. Olson $\sqcup$, Rose JE: Effect of Toxocara canis infection on the ability of white rats to solve maze problems. Exp Parasitol 1966, 19:77-84.

46. Fok E, Szatmari V, Busak K, Rozgonyi F: Prevalence of intestinal parasites in dogs in some urban and rural areas of Hungary. Vet Q 2001, 23:96-98.

47. Lee AC, Schantz PM, Kazacos KR, Montgomery SP, Bowman DD: Epidemiologic and zoonotic aspects of ascarid infections in dogs and cats. Trends Parasitol 2010, 26:155-161.

48. Overgaauw PAM: Prevalence of intestinal nematodes of dogs and cats in the Netherlands. Vet Q 1997, 19:14-17.

49. Parsons JC: Ascarid infections of cats and dogs. Vet Clin North Am Small Anim Pract 1987, 17:1307-1339.

50. Ito K, Sakai K, Okajima T, Quchi K, Funakoshi A, Nishimura J, Ibayashi H, Tsuji $\mathrm{M}$ : Three cases of visceral larva migrans due to ingestion of raw chicken or cow liver. Nippon Naika Gakkai Zasshi 1986, 75:759-766.

51. Nagakura K, Tachibana H, Kaneda Y, Kato Y: Toxocariasis possibly caused by ingesting raw chicken. J Infect Dis 1989, 160:735-736.

52. Sasmal NK, Acharya S, Laha R: Larval migration of Toxocara canis in piglets and transfer of larvae from infected porcine tissue to mice. $J$ Helminthol 2008, 82:245-249.
53. Sommerfelt IE, Rosa A, Duchene A, Degregorio O, Lopez C, Pisanu A, De Torres R: Toxocara canis in experimentally infected pigs: migratory pattern and tissue lesions. Vet Parasitol 2004, 125:323-334.

54. Taira K, Saitoh Y, Okada N, Sugiyama H, Kapel CM: Tolerance to low temperatures of Toxocara cati larvae in chicken muscle tissue. Vet Parasitol 2012, 189:383-386.

55. Taira K, Saeed I, Permin A, Kapel CM: Zoonotic risk of Toxocara canis infection through consumption of pig or poultry viscera. Vet Parasitol 2004, 121:115-124.

doi:10.1186/1756-3305-7-194

Cite this article as: Janecek et al:: Neurotoxocarosis: marked preference of Toxocara canis for the cerebrum and $T$. cati for the cerebellum in the paratenic model host mouse. Parasites \& Vectors 2014 7:194.

\section{Submit your next manuscript to BioMed Central and take full advantage of:}

- Convenient online submission

- Thorough peer review

- No space constraints or color figure charges

- Immediate publication on acceptance

- Inclusion in PubMed, CAS, Scopus and Google Scholar

- Research which is freely available for redistribution

Submit your manuscript at www.biomedcentral.com/submit
C Biomed Central 